\title{
Optimization of EC Values of Nutrient Solution for Tomato Fruits Quality in Hydroponics System Using Artificial Neural Network and Genetic Algorithms
}

\author{
Herry Suhardiyanto ${ }^{1}$, Chusnul Arif ${ }^{2} \&$ Budi I. Setiawan ${ }^{3}$ \\ ${ }^{1,2,3}$ Department of Agricultural Engineering, Bogor Agricultural University, Indonesia \\ ${ }^{1}$ Email: herrysuhardiyanto@ipb.ac.id \\ ${ }^{2}$ Email: chusnul_ar@yahoo.com \\ ${ }^{3}$ Email: budindra@ipb.ac.id
}

\begin{abstract}
Total soluble solids (TSS) and fruit fresh weight are two indicators to show the quality of tomato fruits. To gain high values of TSS and fruit fresh weight, it is important to consider the concentration of nutrient solution, which is commonly represented by Electrical Conductivity (EC) value. Generally, the increasing of EC value not only increases the number of TSS, but also decreases fruit fresh weight. Therefore, it is important to optimize the EC value for both indicators of quality of tomato fruits. The objective of this research is to optimize the EC value of nutrient solution on each generative stage using Artificial Neural Network (ANN) and Genetic Algorithms (GA). ANN was used to identify the relationship between different EC value treatments with TSS value and fruit fresh weight. GA was applied to determine the optimal EC value in generative growth, which is divided into three stages. Results showed that the optimal EC values in the flowering stage, the fruiting stage and the harvesting stage were 1.4 $\mathrm{mS} / \mathrm{cm}, 10.2 \mathrm{mS} / \mathrm{cm}$ and $9.7 \mathrm{mS} / \mathrm{cm}$, respectively.
\end{abstract}

Keywords: artificial neural network; genetic algorithm; hydroponics; tomato fruits quality.

\section{$1 \quad$ Introduction}

Tomato fruit quality can be determined by the following parameters: soluble solid, fresh weight, fruit size, acidity, dry matter, stiffness and flavor. Soluble solids content and fruit fresh weight are the most important indicators for determining tomato fruits quality. Soluble solid content indicates the total solid in fresh tomato fruit soluble in water. It is commonly represented by total soluble solids (TSS) and measured using a refractometer on weight basis (\% brix). Fruit fresh weight is usually affected by its water content. The higher the weight of fresh tomato, the higher the water content is. TSS value and fruit fresh weight are factors related to each other. An increase value in one factor is always associated with a decrease in the other, vise-verse. Both parameters are affected by the concentration of nutrient solution which is commonly represented by electrical conductivity (EC).

ReceivedJune $9^{\text {th }}, 2008$, Revised September $24^{\text {th }}, 2008$, Accepted for publication December $1^{\text {st }}, 2008$. 
Previous studies showed that increasing the concentration of nutrient solution could improve tomato fruit quality by the increase in the number of TSS [1-4]. Unfortunately, as described above, the fruit fresh weight would be decreased [5]. However, excessively high EC value of nutrient solution was associated with poor plant growth. When it was too low, plant growth was stunted from lack of fertilizer [6]. Therefore, it is important to determine the optimum EC value in order to produce tomato fruit with high TSS value and high fruit fresh weight. Accordingly, tomato fruit can be produced with high quality and economic value as desired.

This experiment was conducted with the objective to optimize the EC value in tomato crop cultivation. It was important to identify the relationships between different treatments of EC values and both TSS value as well as fruit fresh weight. Since it was difficult to explain those relationships in mathematical models due to the complexity of the physical and physiological processes involved, another approach was used. Artificial Neural Network (ANN) program is usually suitable for use in dealing with complex systems, such as cultivation system, than that of traditional mathematical methods [7]. ANN has the capability to identify an unknown complex dynamic system [8]. The benefits of using ANN program treatments are due to first its massively parallel distributed structure, and second, its ability to learn and therefore generalize [9]. In addition to the ANN program, Genetic Algorithms (GA) as a powerful tool for use in an optimization process was also used. GA is a combinatorial optimization technique capable of dealing with a complex objective function and simulates the biological evolutionary process based on crossover and mutation in genetics [10].

\section{Materials and Method}

\subsection{Experimental Set Up}

The experiment was conducted in a hydroponics system inside a greenhouse located in Agricultural and Forestry Research Center, University of Tsukuba. The study was focused to gain high TSS value and fruit fresh weight of tomato fruits. Therefore, the optimization process was conducted only in the generative growth phase.

The generative growth was divided into three stages; (1) flowering, (2) fruiting, (3) harvesting. In all stages, the treatments were consisted of two different EC values and two different planting densities (Figure 1). Therefore, there were four patterns of treatments in this experiment. Morimoto et al., [11] found that three or more data sets were necessary for identification using ANN. The EC value treatments consisted of low EC level (range 1,2-2,4 $\mathrm{mS} / \mathrm{cm}$ ) and high EC 
level (range 8,0-10,2 mS/cm). The planting densities consisted of $25 \mathrm{~cm}$ and 10 $\mathrm{cm}$ plant spacing, respectively.

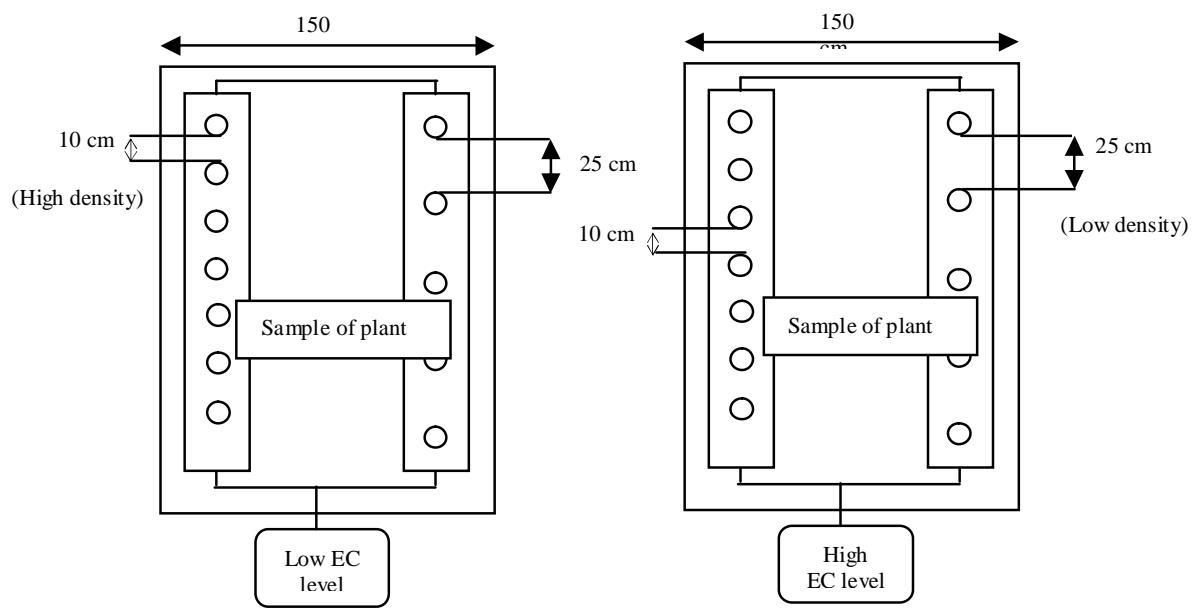

Figure 1 Schematic diagram of experiment.

\subsection{Materials}

Tomato (Lycopersicum esculentum, Mill) cultivar 'Money Maker' seeds were sown in trays with moist vermiculite in a greenhouse. When the cotyledons were fully open, the seedlings were transplanted into rockwool cubes $\left(125 \mathrm{~cm}^{3}\right.$, Nittobo Co., Ltd., Japan) and grown in deep flow technique (DFT) system with Otsuka-A nutrient solution (Otsuka Chemical Co., Ltd., Osaka, Japan). In this stage, the nutrient solution was adjusted to an EC of $1.2 \mathrm{mS} / \mathrm{cm}$ and $\mathrm{pH}$ of 6.5 7.0. After 1 month, the seedlings were transplanted to nutrient film technique (NFT) hydroponics system. Otsuka-B nutrient solution was used in the NFT system in greenhouse. It was supplied to tomato plants in all treatments. When the first flower was opened on the first truss of each plant, pollination was promoted by a vibrator and spraying of 2-methyl-4-chlorophenoxyacetic acid (4-CPA). And then the plants were pinched above so that each plant only had one truss.

\subsection{Development of Artificial Neural Network (ANN) model}

ANN was used to predict the number of TSS and fruit fresh weight. In this study, the ANN model consisted of three layers: input layer, hidden layer and output layer. Some studies [11-14] showed that a three-layer neural network with one hidden layer have gained successful results. Light intensity, plant 
spacing, $\mathrm{EC}$ value in flowering stage $\left(\mathrm{EC}_{1}\right), \mathrm{EC}$ value in fruiting stage $\left(\mathrm{EC}_{2}\right)$ and $\mathrm{EC}$ value in harvesting stage $\left(\mathrm{EC}_{3}\right)$ were inputted, while TSS value and fruit fresh weight were used as the value of the output layer (Figure 2).

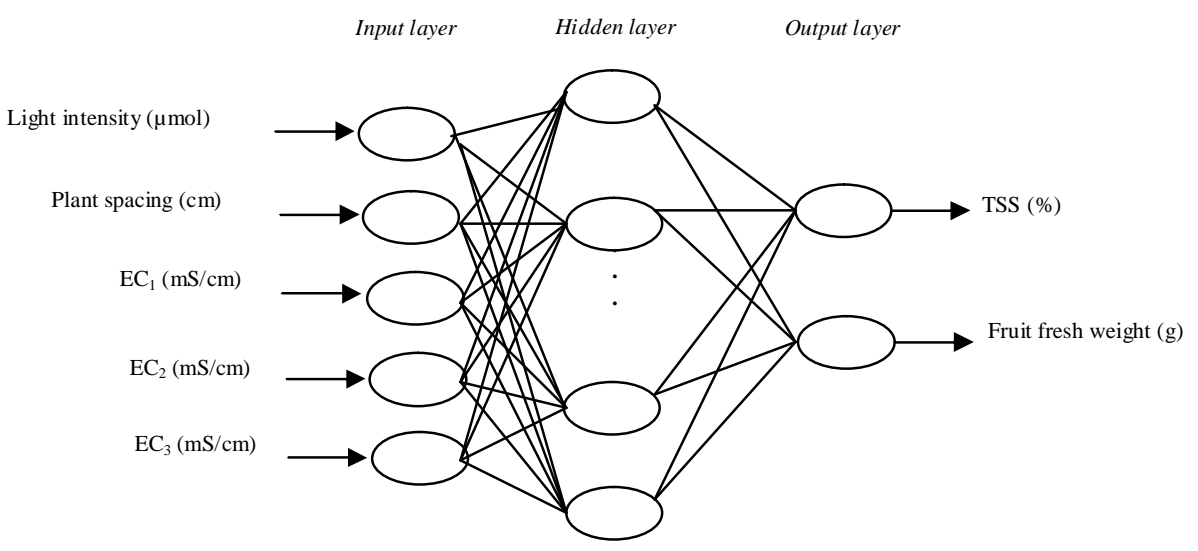

Figure 2 Structure of ANN model.

Back-propagation was used as the learning method, which is composed of two procedures: feed-forward and back-propagation weight trainings. A sigmoid function was selected as the activation function for both hidden layer and output layer. This function has a lengthy history in neural network applications [15], which is given by:

$$
f(n e t)=\frac{1}{1+e^{-n e t}}
$$

where,

$$
n e t=\sum_{i=0}^{n} x_{i} w_{i}
$$

$x_{i}, w_{i}, n$ are the inputs, weights and number of inputs, respectively.

The data samples were divided into two data sets, a training data set and a testing data set. A training data set was used for training the neural network, and a testing data set was used for evaluating the accuracy of the identified model. This type of model validation was called "cross-validation" [16]. The total a training data set was $65 \%$ of all data. The training process resulted weight values of the ANN model for the relationship between input and output parameters. All variables were normalized between 0 and 1, using fixed minimum and maximum values both in training and testing processes. 


\subsection{Development of Genetic Algorithms (GA) model}

GA was used to determine the optimal EC value in flowering, fruiting and harvesting stages. The optimization process was done separately with the ANN model development. The weight values from ANN training were used as input for GA model to predict TSS and fruit fresh weight. Also, $\mathrm{EC}$ values $\left(\mathrm{EC}_{1}, \mathrm{EC}_{2}\right.$, and $\mathrm{EC}_{3}$ ) were used as input for $\mathrm{GA}$ model. The $\mathrm{EC}$ values were determined through searching method in GA model.

The objective function was given by the sum of the average value of TSS and fruit fresh weight on each stage (Equation 3). The problem of this summation was the different units of TSS value and fruit fresh weight. To avoid the problem, both parameters were normalized between 0.2 and 0.8 .

$$
F\left(E C_{1}, E C_{2}, E C_{3}\right)=\frac{1}{m}\left(\sum_{j=1}^{n} \sum_{i=1}^{m} \frac{T S S_{i j}}{m}+\sum_{j=1}^{n} \sum_{i=1}^{m} \frac{W_{i j}}{m}\right)
$$

Maximize $F\left(E C_{1}, E C_{2}, E C_{3}\right)$

Subject to $1,4 \leq E C_{1} \leq 9,6 ; 2,2 \leq E C_{2} \leq 10,2 ; 2,1 \leq E C_{3} \leq 9,7 \mathrm{mS} / \mathrm{cm}$

where

$F\left(E C_{1}, E C_{2}, E C_{3}\right)$ : function of nutrient concentration, dimensionless.

TSS : Total soluble solid (\%)

$W \quad$ : Fruit fresh weight $(\mathrm{g})$

$m \quad$ : number of plants

$n \quad:$ number of treatments

In order to employ $G A$, individual, fitness and $G A$ operators were defined as follows:

1. Definition of the individual:

In the $G A$ optimization, an individual represented a candidate for the optimal solution. In this problem, the optimal $E C$ value in flowering, fruiting and harvesting stages were determined. Therefore, the three optimal values of nutrient concentration, $E C_{1}, E C_{2}, E C_{3}$ represented an individual and each EC representative was coded as six-bit binary string. As an illustration, an individual was given as follow:

$$
\begin{aligned}
\text { Individual } & =E C_{1}, E C_{2}, E C_{3} \\
& =011000,101001,001010 \text { (binary string) } \\
& =4.52,7.40,3.30 \text { (decimal values) }
\end{aligned}
$$

A set of individual was called a population. They evolved toward better solution. $G A$ worked with a population involving many individuals. 
2. Fitness function:

Fitness function was an indicator to show the quality of an individual. All of individuals in population were evaluated in terms of their performances by this function. The higher fitness functions of an individual, the better the ability to survive. In this problem, i.e., maximizing problem, fitness function was given as same as objective function (Equation 3).

3. $G A$ operators

The main operators were crossover and mutation. Crossover combined features from two individuals based on crossover rate $(\mathrm{Pc})$. It operated by swapping corresponding component in the binary strings representing an individual. Here, one-point crossover was used in each parameter of EC. Mutation inverted one or more bits binary string (also called gene) in each individual based on mutation rate from 0 to 1 or 1 to 0 .

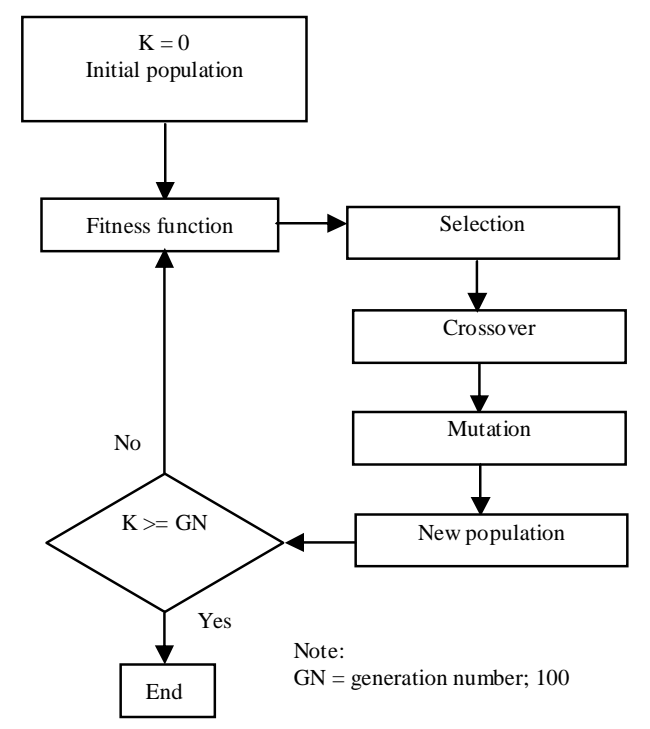

Figure 3 Flowchart of optimization process using GA.

Procedures of optimization using GA can be seen in Figure 3 and could be further explained as follows:

a) An initial population consisting of ten individuals was generated at random.

b) The fitness values of all individuals were calculated using Equation 3. 
c) The performance of each individual was evaluated using the elitism strategy, i.e., $60 \%$ of all individuals with highest performance was selected and retained for next generation.

d) Crossover and mutation operations were applied to the selected individuals.

e) The new population was created.

f) Steps (b) to (d) were repeated until the required generation number achieved. An optimal value was given as an individual with highest fitness.

\section{$3 \quad$ Results and Discussion}

\subsection{Identification Process by using ANN}

The training process was carried out using the following ANN parameters: learning rate $=0.6$, momentum value $=0.6$ with total iteration of 1000 and seven nodes of hidden layer. Figure 4 shows the identification results in the response of TSS value and fruit fresh weight to the EC values, plant spacing and light intensity. The data used in the test was independent from the training data.
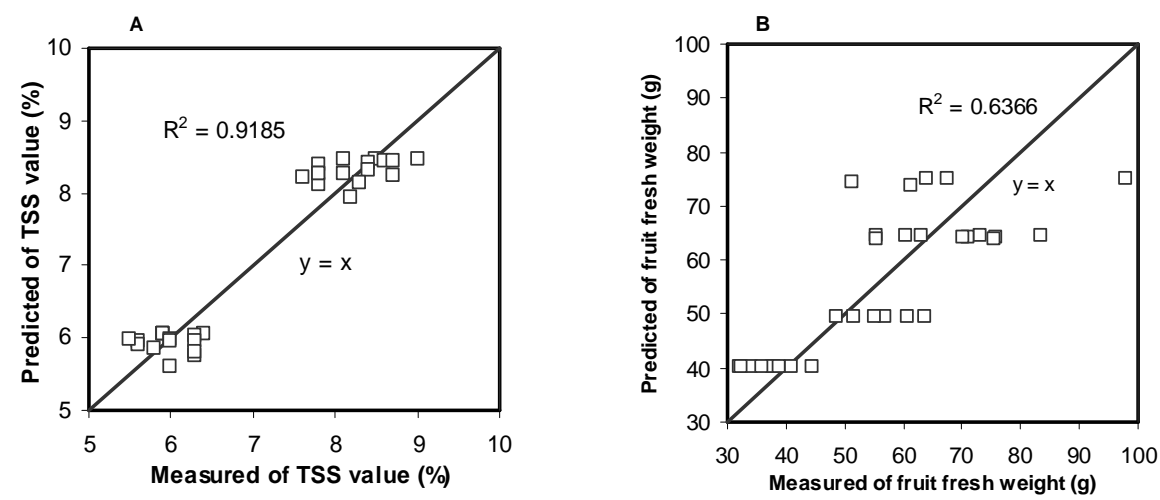

Figure 4 Comparison between measured data and predicted data by ANN models; A: TSS value; B: fruit fresh weight.

It can be seen in Figure 4 that the coefficients of determination $\left(\mathrm{R}^{2}\right)$ values for TSS value and fruit fresh weight are 0.9185 and 0.6366 , respectively. Overall, it was found that the predicted data were closely related to measured data. This result means that a reliable computational model could be obtained for predicting TSS value and fruit fresh weight. 


\subsection{Optimization Process by using GA}

As explained in the previous section, GA was used to determine the optimal EC value in flowering $\left(\mathrm{EC}_{1}\right)$, fruiting $\left(\mathrm{EC}_{2}\right)$ and harvesting $\left(\mathrm{EC}_{3}\right)$ stages. GA searched the optimal $\mathrm{EC}$ value by interval $1.4 \leq \mathrm{EC}_{1} \leq 9.6 ; 2,2 \leq \mathrm{EC}_{2} \leq 10.2$; $2.1 \leq \mathrm{EC}_{3} \leq 9.7 \mathrm{mS} / \mathrm{cm}$. GA operators (crossover and mutation rate) are important factor for the algorithm to perform the optimization satisfactorily. The crossover rate $(\mathrm{Pc})$ was selected to be 0.6 , while mutation rate $(\mathrm{Pm})$ was selected to be 0.05 , respectively. By those values, expectedly, both the diversity of individuals and a global optimum can be reached.

Figure 5 shows the evolution curves during the search for an optimal value of the fitness. The fitness value increased sharply from the first to $12^{\text {th }}$ generation and reached the maximum value at $22^{\text {nd }}$ generation. From $12^{\text {th }}$ to $22^{\text {nd }}$ generation, the fitness value increased gradually from 1.0563 to 1.0604 . After $22^{\text {nd }}$ generation, the fitness value reached its constant value (1.0604).

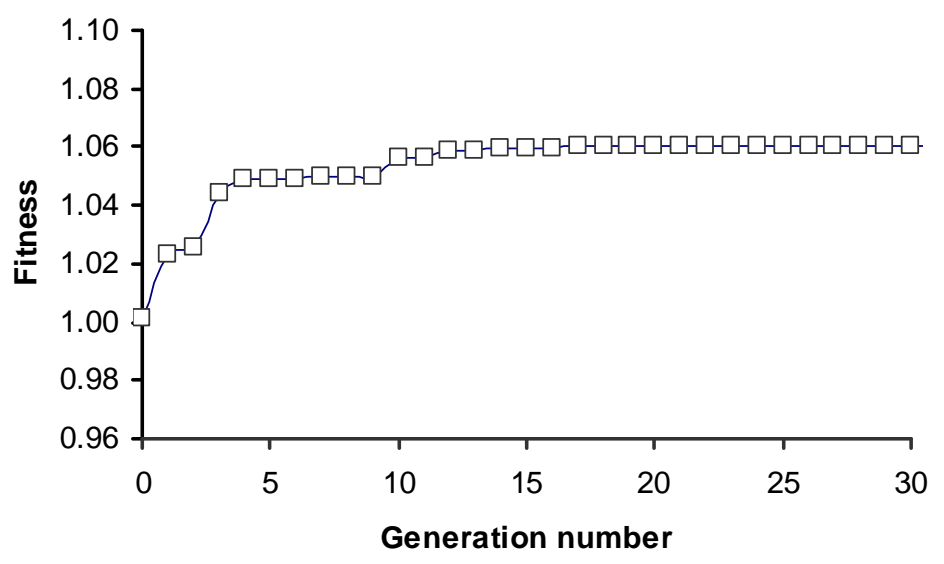

Figure 5 Evolution curves in searching for an optimal value of fitness function. 


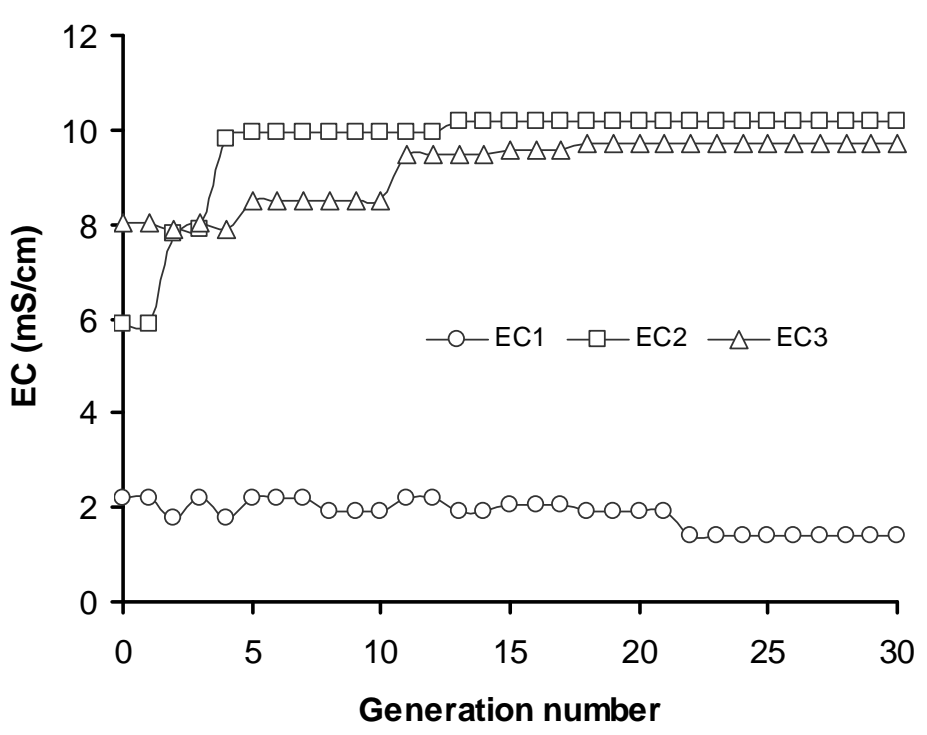

Figure 6 Evaluation curves in searching the optimal EC values on each generative growth stage.

Figure 6 shows the evolution curves during the search for the optimal EC values on each generative stage. GA optimizer recommended maintaining a markedly lower level $(1.4 \mathrm{mS} / \mathrm{cm})$ at flowering stage $\left(\mathrm{EC}_{1}\right)$, and a slightly high level at both fruiting and harvesting stages. The optimal EC values were 10.2 and 9.7 $\mathrm{mS} / \mathrm{cm}$ at the fruiting and harvesting stage, respectively. In hydroponics system, it is known that the concentration of nutrient solution is one of the most important control factors for adjusting the balance between the vegetative and generative growth [17]. In the vegetative growth, as explained previously, plants focus on the development of root, stem and leaf. Therefore, maximum water uptake by root is needed. However, the low EC value is essential for the plants. The flowering stage is the first stage after vegetative stage. In this stage, the low EC value could be effective in suppressing the excessive vegetative growth. On the other hand, the high EC value in the fruiting and harvesting stages appears to be useful in accelerating generative growth. By this reason, however, the result of GA optimization clearly maximized fitness. 

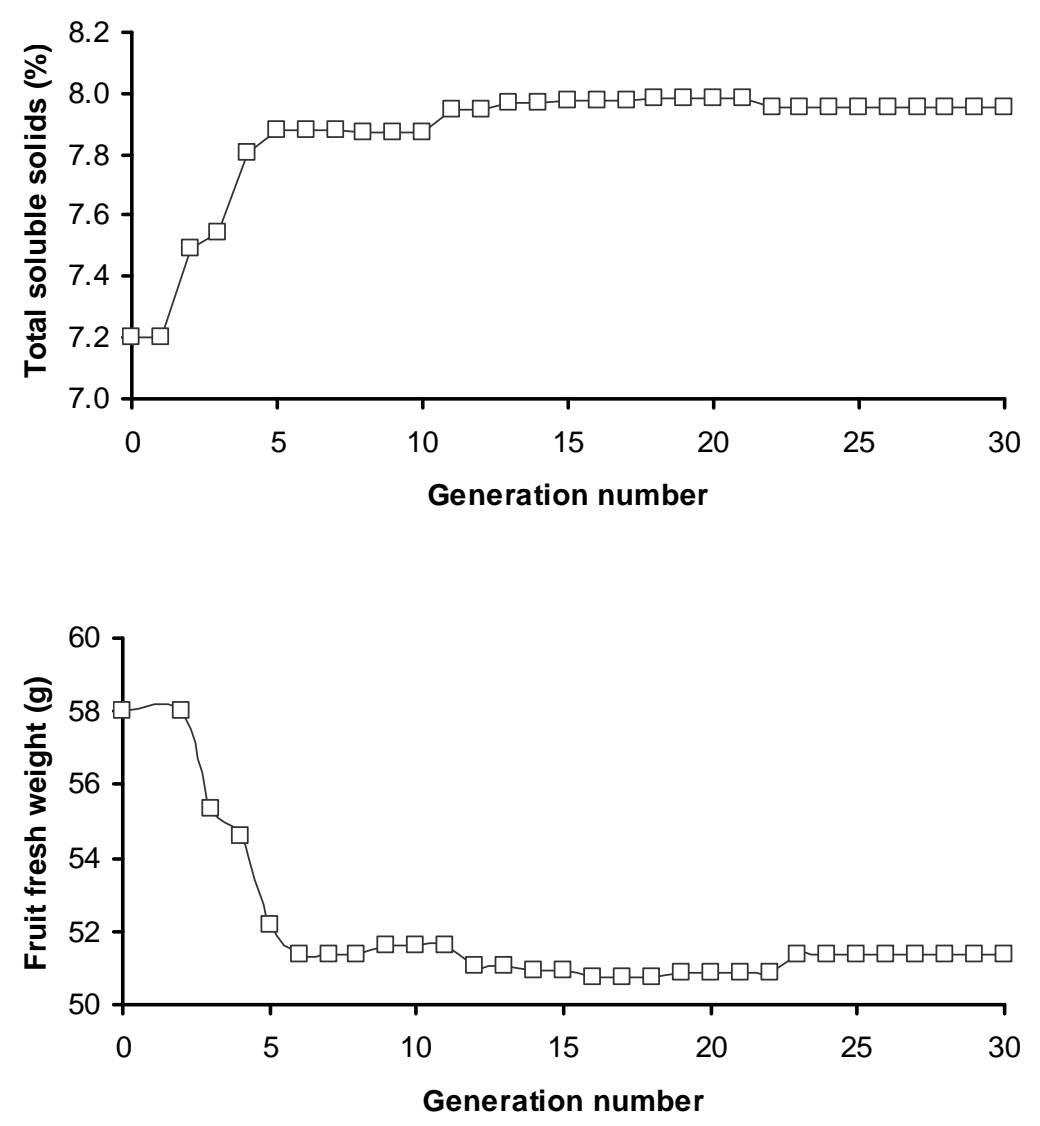

Figure 7 The estimated TSS value and fruit fresh weight to the optimal EC value.

Figure 7 shows the estimated TSS value and fruit fresh weight results calculated through the simulation using ANN model. The upper figure is the estimated TSS value of tomato fruit. The lower figure is the estimated fruit fresh weight of tomato fruit. By the optimal EC value, it was estimated that the results of TSS value and fruit fresh weight were $7.9 \%$ and $51.34 \mathrm{~g}$, respectively. These results indicated good quality of tomato fruit suitable to be produced. 


\section{Conclusion}

A computer program for optimization of EC values of nutrient solution on hydroponics system was developed using Artificial Neural Network (ANN) and Genetic Algorithms (GA). ANN was used to identify the relationship between differences EC value treatments with TSS value and fruit fresh weight. GA was applied to determine the optimal EC value. The ANN model consisted of three layers with seven nodes of hidden layers. The results indicated that the predicted data were closely related to measured data. GA model successfully searched the optimal EC values in flowering, fruiting and harvesting stages that were for $1.4 \mathrm{mS} / \mathrm{cm}, 10.2 \mathrm{mS} / \mathrm{cm}$ and $9.7 \mathrm{mS} / \mathrm{cm}$, respectively.

\section{References}

[1] Adams, P. \& Ho, L.C., Effect of constant and fluctuating salinity on yield, quality and calcium status of tomatoes, J. Hort. Sci., 64, pp. 725-732, 1989.

[2] Adams, P., Effect of increasing the salinity of the nutrient solution with major nutrients or sodium chloride on the yield, quality and composition of tomatoes grown in rockwool, J. Hort. Sci., 66, pp. 201 - 207, 1991.

[3] Sanchez, A. R, Romero-Aranda, R., Cuartero, J., Plant water uptake and water use efficiency of greenhouse tomato cultivars irrigated with saline water, Agric. Water Management, 78, pp. 54-66, 2005.

[4] Saito, T., Fukuda, Naoya \& Nishimura, Shigeo, Effect of Salinity Treatment Duration and Planting Density on Size and Sugar Content of Hydroponically Grown Tomato Fruits. Journal Japan, Soc. Hort. Sci., 75 (5), pp. 392-398, 2006.

[5] Li, Y. L., Stanghellini, C. \& Challa, H., Effect of electrical conductivity and transpiration on production of greenhouse tomato (Lycopersicum esculentum L.), Sci. Hort, 88, pp. 11-29, 2001.

[6] Whipker, B. E. \& Cavins, Todd J., Electrical Conductivity (EC): Unit and Conversions, NCSU Floriculture Research Report 002. http://www.ces.ncsu.edu/depts/hort/floriculture/Florex/EC\%20Conversio n.pdf. December 2000.

[7] Hashimoto, Y., Application artificial neural network and genetic algorithms to agricultural systems, Computer and Electronics in Agriculture, (18), pp. 71-72, 1997.

[8] Purwar, S.I.N. Kar \& Jha, A.N., On-line system identification of complex system using Chebyshev neural networks, Applied Soft Computing, (7), pp. 364-372, 2007.

[9] Nugroho, A. Satrio, Information Analysis Using Soft Computing - The Applications to Character Recognition, Meteorological Prediction and 
Bioinformatic Problems, $\mathrm{PhD}$ Dissertation, Nagoya Institute of Technology, Nagoya, Japan, pp. 12, 2003.

[10] Goldberg, D.E., Genetic Algorithms in Search, Optimization, and Machine Learning, Addison-Wesley, Reading, Massachusetts, pp. 412, 1989.

[11] Morimoto, T., Purwanto, W., Suzuki, J. \& Hashimoto, Y., Optimization of heat treatment for fruit during storage using neural network and genetic algorithms, Computers and electronics in agriculture, (19), pp. 87 - 101, 1997.

[12] Linker, R, \& Seginer, I. \& Gutman, P.O., Optimal $\mathrm{CO}_{2}$ control in a greenhouse modeled with neural networks, Computers and electronics in agriculture, (19), pp. 289-310, 1998.

[13] Kondo, N., Ahmad, Usman, Monta, Mitsuji \& Murase, Haruhiko, Machine vision based quality evaluation of Iyokan orange fruit using neural networks, Computers and electronics in agriculture, (29), 135-147, 2000.

[14] Qiao, J, Wang, N., Ngadi, M.O. \& Kazemi, S., Predicting mechanical properties of fried chicken nuggets using image processing and neural network technique, Journal of Food Engineering, (79), pp. 1065 - 1070, 2007.

[15] Kros, John F., Lin, Mike \& L. Brown, Marvin, Effect of neural network sSigmoid function on KDD in the presence of imprecise data, Computer \& Operation Research, (33), 3136-3149, 2006.

[16] Morimoto, T. \& Y. Hashimoto, AI approaches to identification and control of total plant production systems, Control Engineering Practice, (8), 555-567, 2000.

[17] Ehret, D. \& Ho, L.C., Effects of osmotic potential in nutrient solution on diurnal growth of tomato fruit, Journal of Experimental Botany 37(182), pp. 1294-1302, 1986. 